\title{
Activities Recognition and Worker Profiling in the Intelligent Office Environment Using a Fuzzy Finite State Machine
}

\author{
Caroline Langensiepen, Ahmad Lotfi and Saifullizam Puteh
}

\begin{abstract}
Analysis of the office workers' activities of daily working in an intelligent office environment can be used to optimize energy consumption and also office workers' comfort. To achieve this end, it is essential to recognise office workers' activities including short breaks, meetings and non-computer activities to allow an optimum control strategy to be implemented. In this paper, fuzzy finite state machines are used to model an office worker's behaviour. The model will incorporate sensory data collected from the environment as the input and some pre-defined fuzzy states are used to develop the model. Experimental results are presented to illustrate the effectiveness of this approach. The activity models of different individual workers as inferred from the sensory devices can be distinguished. However, further investigation is required to create a more complete model.
\end{abstract}

Index Terms-ubiquitous and pervasive computing, ambient intelligence, fuzzy finite state machines, intelligent environments, intelligent offices, energy saving.

\section{INTRODUCTION}

An office environment equipped with appropriate sensory devices and actuators is referred to as an "Intelligent Office" environment. Analysis of the office workers' Activities of Daily Working (ADW) in an intelligent office environment can be used to optimize energy consumption and also office workers' comfort. Therefore it is essential to identify individual worker profiles based on the worker's preferences and behaviour. The individual profile will then be used to automatically adjust office conditions according to the worker preferences. Sensory signal outputs from a monitoring system can be used to recognise activities and ultimately create a profile for each worker.

In modern office environments, lighting systems, heating/cooling systems and PCs are the main energy consumers. Many companies would like to reduce their energy usage for two reasons. The first is that they worry about the environment and want to reduce the impact they have on it. The second reason, and most likely the one that companies care about most, is cost.

Caroline Langensiepen and Ahmad Lotfi are with School of Science and Technology, Nottingham Trent University, Nottingham, United Kingdom (email: \{caroline.langensiepen,ahmad.lotfi\}@ntu.ac.uk). Saifullizam Puteh is with Universiti Tun Hussein Onn Malaysia (UTHM), Malaysia.
For example, PCs waste a lot of energy due to being left on for long periods of time when not in use. Even though they have power management modes to reduce energy consumptions when they are not in use, these are not always applied in a reasonable way.

Some workplaces incorporate reactive systems such as Passive Infra-red (PIR) activated lighting, but these can be activated/deactivated inappropriately. Heating systems often work on the assumption of a 9:00 AM to 5:00 PM presence, five days a week, whereas an individual office worker may have a different schedule, including long periods out of the office. Similarly, automated office computer shut down may be set based on assumptions of behaviour that are inappropriate, leading workers to try to find ways of subverting the mechanisms so that their computer remains on and avoids the inconvenience of a slow restart.

The aim of the research presented in this paper is to investigate ways of improving the energy efficiency in an intelligent office environment taking into account worker preferences. This is achieved by adapting the environment to the worker profile needs and requirements. If the energy consumption units including lighting, heating and $\mathrm{PC}$ are made more responsive to the worker's habits, routines and preferences, there would be more acceptance of their use. The research challenge is to generate such profiles based on data gathered from the sensory devices installed in the intelligent office environment.

To establish a relationship between the data collected from sensors and a representation of the behaviour of the office worker, Fuzzy Finite State Machines (FFSM) are used to model the behaviour. Different activities are presented as different states in FFSM.

This paper is organised as follows: in Section II a short summary of the related work regarding ubiquitous computing in office environments is presented. Fuzzy finite state machines are explained in Section III followed by the proposed worker profiling framework in Section IV. The implementation of fuzzy finite state machines for activity recognition is in Section V. Some experimental results are presented in Section VI. Section VII draws some conclusions and discusses the direction in which the research needs to progress. 


\section{RELATED WORK}

Human activities recognition in buildings is a subject of interest for many researchers. Activities recognition in home environments [1] and the office environment [2] are currently being investigated. For example, the authors in [3] have conducted research based on sensorbased human activity recognition. They suggested a complex process of activity recognition that can be approximately classified by four basic steps. Recommended steps are: a) to choose and deploy appropriate sensors in order to monitor and capture worker's behaviour in buildings, b) to collect, store, and process perceived information for data representation at an appropriate level, c) information gathered from monitoring locations based on activities of daily living are used to create computational activity models, and d) to select and develop intelligent algorithms to infer activities from sensory data.

In [4] the authors examined intermittent activities that interrupt the planned "normal" activities of office workers. They found that probabilistic and S-curve methods could be used to predict activities (such as "smoking", "go to toilet") and these could be used in fine grained simulations of building performance. However, they cautioned that the results applied to typical office based organisations, and other office environments might need further experiments to generate data.

People's activity in a building is based on their schedule of work, lifestyle and social activity. It is mentioned in [5] that one of the key features of an intelligent environment is to provide monitoring of Activities of Daily Living (ADL). In many studies [1], [6], ADL are monitored to assess elderly people's activity in the home environment, and attempts are made to process activity sequences to make them more understandable. For example, daily home activity involves basic functions like preparing breakfast or food, showering, walking, sleeping, watching television, reading books etc.

Recently, advanced intelligent sensor technology has resulted in various types of sensors that have been used by researchers to extract features from activity monitoring. A study in [7] has used physiological sensors (cardiac frequency, activity or agitation, posture and fall detection sensor), microphones, PIR sensors, door sensors and state-change sensors. This monitoring system has been combined with a fuzzy logic system for recognizing activities in readiness for the next generation of smart houses. The authors in [8] have proposed hardware and software design and implementation of lowcost, wearable, and unobtrusive intelligent sensors for monitoring human physical activities. Many researchers have successfully conducted research with similar ideas

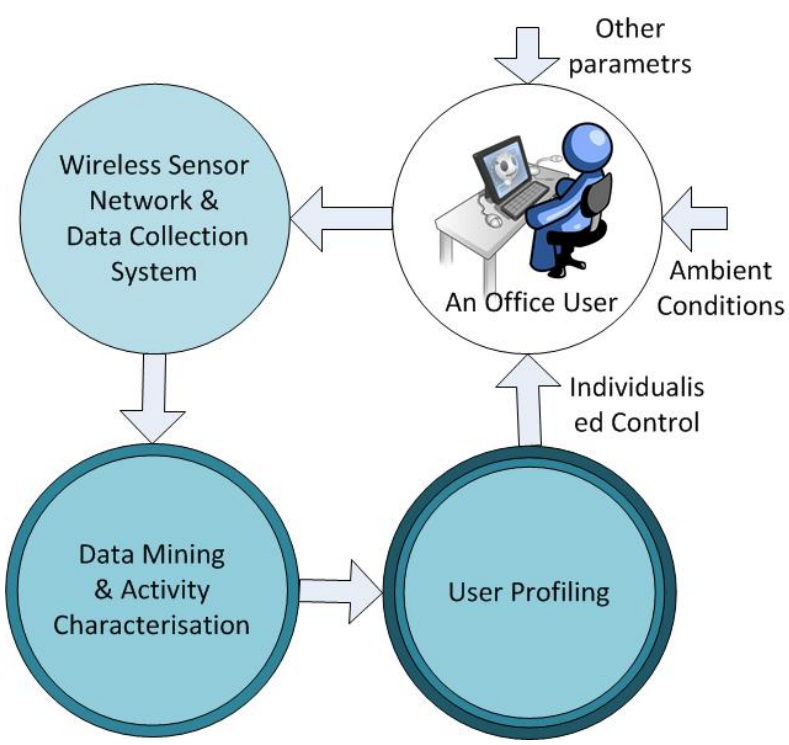

Fig. 1: Proposed framework for worker profiling in the intelligent office environment.

in activity monitoring systems, such as Interactive Continuous Autonomic Logging and Monitoring (iCALM) [9], human activity recognition in pervasive health-care systems and occupancy monitoring systems [10].

To recognise human activities, different computational intelligence techniques have been applied. Data mining techniques including Discontinuous VariedOrder Sequential Miner [11], classification tree methods [12] and Hidden Markov Models (HMMs) have been used [13]. More intelligent computational techniques including Fuzzy Inference Systems in activity detection [14] and some hybrid soft computing approaches namely neuro-fuzzy techniques [15], [16] have also been investigated. Some statistical methods and Bayesian classifiers are discussed in [17] and [18].

\section{FuZZy Finite State Machines}

A Finite-State Machine (FSM) is used to model sequential events and activities. To improve the performance of FSM, a Fuzzy Finite-State Machine (FFSM) is proposed where transitions are not triggered by crisp events but by fuzzy variables, and state transitions are fuzzy as well. Therefore, at any time, the whole system is not necessarily in one and only one state, but it may be in more states at the same time, each one associated with its own membership value [19].

For a non-sequential system, the state at time $t$ is presented as state variables $X(t)=\left[x_{1}, x_{2}, \ldots, x_{n}\right]$. When the state of the system evolves in time to the next state $X(t+1)$, the general form of the time-invariant 
model is formulated by the following set of equations:

$$
\begin{aligned}
X(t+1) & =f(X(t), U(t)) \\
Y(t) & =g(X(t), U(t))
\end{aligned}
$$

where $U(t)=\left[u_{1}, u_{2}, \ldots, u_{p}\right]$ is the input vector, $Y(t)=\left[y_{1}, y_{2}, \ldots, y_{q}\right]$ is the output vector, $f$ is the function that calculates the state vector $S$ at time step $t+1$, and $g$ is the function that calculates the output vector $Y$ at time step $t . n, p$ and $q$ are number of states, input variables and output variables respectively.The initial state is also presented as $X(t=0)=X_{0}$.

For many systems, due to their complexity, it is impossible to identify functions $f$ and $g$. For a sequential system, this would be even harder to obtain. To overcome the complexity of the modelling, use of FFSM is proposed and it has been proven to be a useful tool for modelling [20], [21]. FFSM is a quintuple $\{X, U, f, Y, g\}$ where $X$ is the set of fuzzy states of the system; $U$ is the set of input vectors; $Y$ is the set of output vectors; $f$ is the transition function for the set of states and $g$ is the output function that calculates the set of output vectors. More details about these components are provided below:

\section{A. Fuzzy States}

Fuzzy states, $X$ is the set of states which is defined as linguistic variables with linguistic labels $\left\{x_{1}, x_{2}, \ldots, x_{n}\right\}$. Fuzzy states of the system are represented via a state membership vector $S(t)=$ $\left[s_{1}, s_{2}, \ldots, s_{n}\right]$, where $\mu_{s_{i}} \in\left[\begin{array}{ll}0 & 1\end{array}\right]$.

\section{B. Input Variables}

The input vector $U=\left[u_{1}, u_{2}, \ldots, u_{p}\right]$ is a set of linguistic variables obtained from the the sensors. Linguistic values associated with inputs are represented as $A_{u_{i}}=\left\{A_{u_{i}}^{1}, A_{u_{i}}^{2}, \ldots, A_{u_{i}}^{p_{i}}\right\}$, where $p_{i}$ is the number of linguistic labels of the linguistic variable $u_{i}$.

\section{Output Variables}

The output vector $Y(t)=\left[y_{1}, y_{2}, \ldots, y_{q}\right]$ is a vector of crisp values. Outputs are calculated based on the current state of the system.

\section{Output Function}

The output function $g$ calculates the value of the output vector $Y(t)$. It could be a simple combination of state activation memberships. Hence the output is the current fuzzy state of the system.

\section{E. Transition Function}

The transition function $f$ will provide the means of calculation of the next value for state activation. This function is implemented by means of a set of fuzzy rules. All relationships are initially defined by an expert. There are ways of improving the expert rules but this is not within the scope of this paper. In general, rules are presented as:

$R_{i j}$ : IF $\left(S(t)\right.$ is $\left.x_{i}\right)$ AND $\Theta_{i j}$ THEN $S(t+1)$ is $x_{j}$ where all constraints imposed on inputs are represented as $\Theta_{i j}$. For example $\Theta_{i j}=\left(u_{1}(t)\right.$ is $\left.A_{u_{1}}^{2}\right)$ AND $\left(u_{2}(t)\right.$ is $\left.A_{u_{1}}^{3}\right)$.

FFSA can be of different types: time-independent and time-dependent. They differ only in the way state activity moves from one state to another, according to the degrees of membership of the fuzzy transitions. For a time-dependent system the rule could be:

$R_{i j}$ : IF $\left(S(t)\right.$ is $\left.x_{i}\right)$ AND $\Theta_{i j}$ THEN $S(t+1)$ flows to $x_{j}$

To calculate the total output of the rules, and therefore the state activation vector $S(t+1)$, a weighted average of all rules based on their firing strength is computed.

\section{WORKER PROFILING FrAMEWORK}

The proposed framework for worker profiling in the intelligent office environment is illustrated in Fig. 1. There are three distinct phases to develop the research; in the first stage, a data collection system is utilised. The data collection system collects environmental conditions, worker activities and office conditions. In the second phase, data mining techniques are applied to identify different worker characteristics. In the third stage, activities recognised from a worker are represented as a worker profile. The worker profile is used to summarise the activities of a worker in an office environment. The worker profile is also used to optimise the environmental control system, so that the office conditions are adjusted in line with the individual worker.

The proposed system architecture to measure office worker's activities and energy usage comprises the following components.

\section{A. Sensors}

The ambient conditions are recorded via temperature, humidity and light sensors (inside the office and external to the building). The responses of the office worker are also captured via his/her activities in the office environment. The most commonly used sensor types in a smart environment are as follows:

- Motion sensor (On/Off)

- Door entry point sensor (On/Off) 
- Windows entry point sensor (On/Off)

- Pressure sensor measuring chair occupancy (On/Off)

- Room temperature

- Room humidity

- Outside temperature

- Room light intensity

- Ambient light intensity

\section{B. Communication System}

To communicate the information gathered from sensors to the data logger system, both wired and wireless communication systems are investigated. In order to make the data collection system more flexible and less intrusive, a Wireless Sensor Network (WSN) is developed. The developed WSN is based on ZigBee wireless technology. The ZigBee network is proven to be a suitable technology for our application mainly because it is a low date rate, low power consumption and low cost wireless standard.

\section{Personal Computer Monitoring Application}

Information regarding the office $\mathrm{PC}$ is collected using a monitoring application agent installed on the PC to be monitored. A monitoring software agent logs keyboard and mouse activities. These activities are recorded in a database along with the rest of the data collected from the WSN.

The PC monitoring application needs to communicate with the control server application in both directions. It needs to send a message to the control server application when the PC has gone idle or change state, informing the control server application that the PC's state has changed.

\section{Office Worker Activity Characterisation Module}

Office worker activity characteristics are those traits that differentiate an office worker from other office workers or the same worker in two different time periods. For example coming to work early or working until the late evening are characteristics of a specific worker that differentiate them from others. Office worker activities and behaviour characterisation will lead to the generation of a profile. The office worker profile is then used to interact with the environment and controlling different elements if necessary. An individual office worker profile is associated with the office worker's work habit and his/her preferences.

\section{A Fuzzy Finite State Machine for Activity RECOGNITION}

The sequence of activities associated with a worker in the workplace represents the pattern of their work.Thus

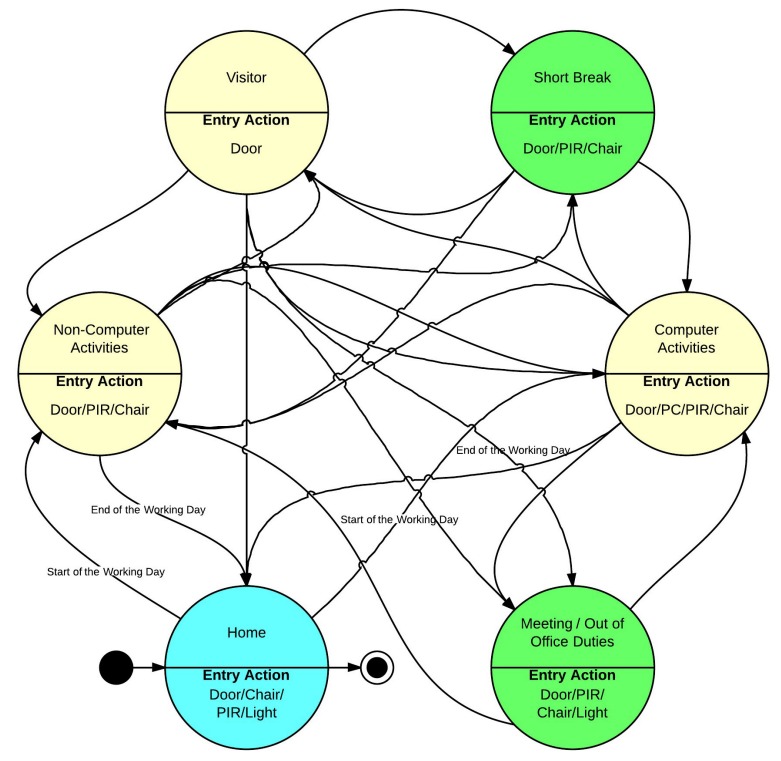

Fig. 2: State diagram of office activities.

activity recognition is an essential element of effective profiling and eventually performing optimum control of the working environment. Recognising complex activities is a challenging and active area of research [22]. Specifically, the nature of human activities presents the following challenges.

- Recognise concurrent activities, where several activities are conducted at the same time. For example, people can work with their computers while they are talking over the phone or reading a book. Therefore, recognising concurrent activities should be dealt with using a different approach from that for simple sequential activity.

- Recognising interleaved activities, since some activities may be interleaved. For example, if the office worker is reading a report and someone enters the office, the first activity will be paused for a while and after the meeting, the first reading activity will be resumed.

- Multiple occupancy - in many office environments more than one office worker may be present in a given office. The activities that are being performed by a specific worker need to be recognised. For example two workers can enter the room at the same time and they will use different areas of the room for their activities.

We propose to map the elements of a fuzzy state machine onto the daily activities of an office worker as follows: 


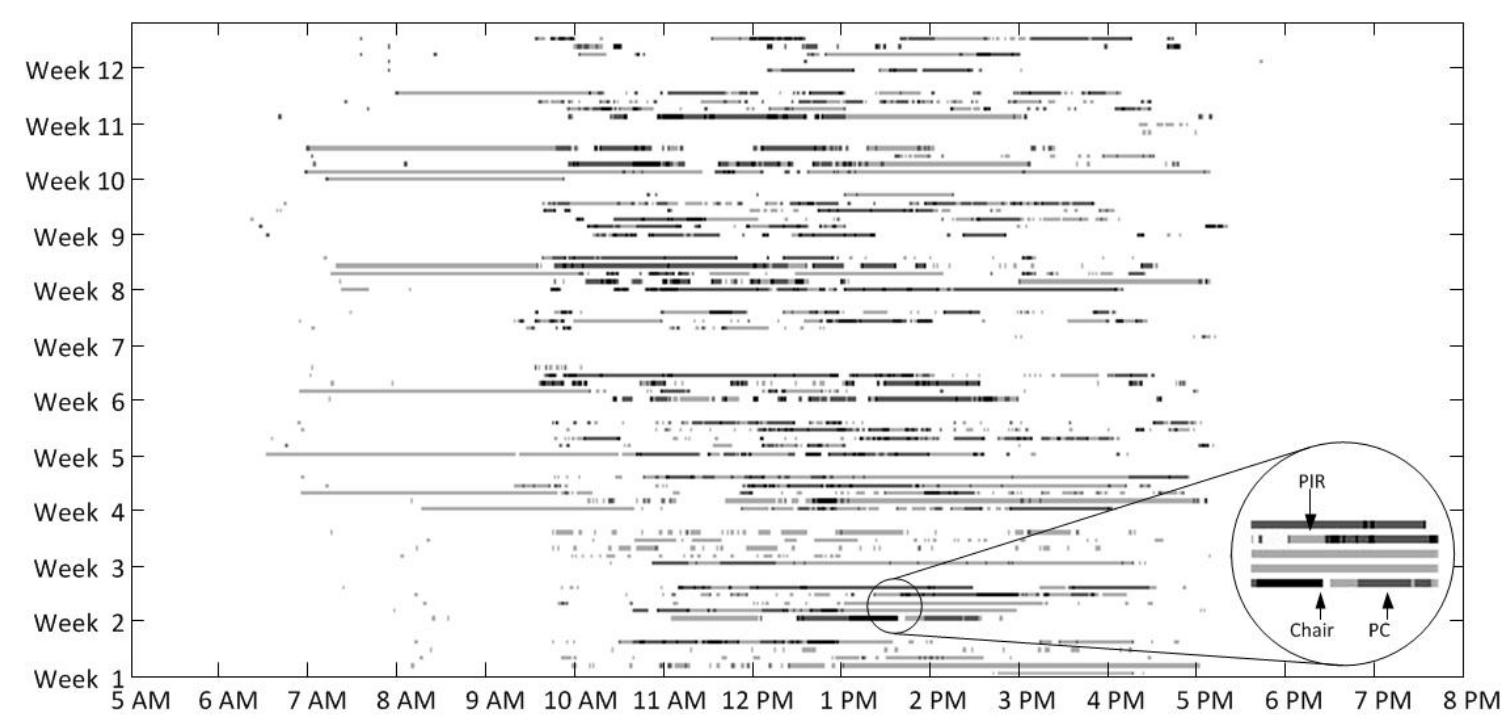

Fig. 3: Sample of activities for worker \#4.

\section{A. Fuzzy States (Activities)}

To recognise activities of an office worker, we have considered 6 fuzzy states representing 6 distinguishable activities. They are:

- $x_{1} \longrightarrow$ Home. At the start of the day and the end of the day, the office worker will be at home. However, the timing is not very exact and it could vary for individual office workers and for different days of the week even for the same individual.

- $x_{2} \longrightarrow$ Meeting and out of office duties. The worker will be out of office during the day and the duration of the meeting will be influenced by other factors which are difficult to measure.

- $x_{3} \longrightarrow$ Short break, where worker may take a few minutes for a comfort break or coffee break.

- $x_{4} \longrightarrow$ Visitor. There will be another person present in the environment and this could affect the other office worker activities.

- $x_{5} \longrightarrow$ Non-computer activities, when a worker is reading or involved in activities where a computer is not needed.

- $x_{6} \longrightarrow$ Computer activities. This is the most common activity in a modern office environment where a computer is used and an office worker is interacting with his/her PC.

A schematic diagram of the proposed state diagram of office activities is shown in Fig. 2.

\section{B. Input Variables}

There are two sets of inputs representing office worker's working behaviour and preferences. Our focus is primarily on the behaviour rather than preferences. Inputs associated with behaviour are:

- $u_{1} \longrightarrow$ Chair occupancy, presented as binary values to show when chair is used or not used.

- $u_{2} \longrightarrow$ Motion sensor and room occupancy. A PIR sensor is used as an indicator of room usage. However this is not sufficient and it should be considered in conjunction with other inputs.

- $u_{3} \longrightarrow$ Door entry point sensor, presented as binary values and activated when entry door is opened.

- $u_{4} \longrightarrow$ Computer usage, keyboard and mouse events and in general an indicator of when a PC is in use.

- $u_{5} \longrightarrow$ Room light intensity is also an indicator of room occupancy, since the office worker will switch their light on/off.

The second group of sensors monitor room temperature, room humidity, windows entry point sensor, outside temperature and ambient light intensity. These will record aspects related to worker comfort and preferences - which as stated above are not our primary focus at present.

\section{Transition Function}

As stated earlier, a state transition is represented as a set of rules. Rules will have the following structure:

$R_{14}$ : IF $\left(S(t)\right.$ is $\left.x_{1}\right)$ AND $\Theta_{14}$ THEN $S(t+1)$ flows to $x_{4}$

$R_{15}$ : IF $\left(S(t)\right.$ is $\left.x_{1}\right)$ AND $\Theta_{15}$ THEN $S(t+1)$ flows to $x_{5}$ 


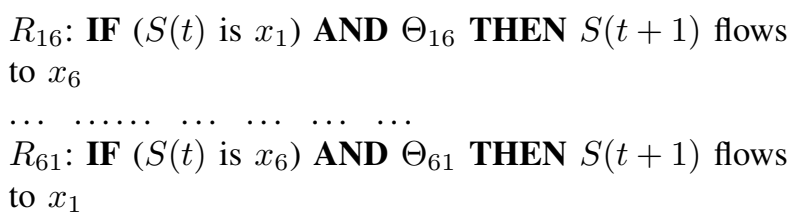

There are some limitations - for example, when a worker is in the home state, $x_{1}$, the model can only transition to $x_{4}, x_{5}$ and $x_{6}$. Based on the information gathered from sensors, it is not possible to infer the transition to $x_{2}$ and $x_{3}$; i.e. going to a meeting or short break states directly from the home state.

\section{Output Variables and Output Function}

Output variables are the same as the states activation membership i.e. $Y(t)=S(t)$.

\section{RESULTS}

The office environment used as the testbed for our experiment is a set of four academic staff offices at the Computing and Informatics Building, Nottingham Trent University. Collected data from the offices include room status, power use of office equipment and ambient information. In total, each office is equipped with 9 sensors measuring different activities and properties of the testbed environment. There are four offices represented as A, B, C and D and also there are four different workers represented as worker \#1, \#2, \#3 and \#4. A sample of activities for worker \#4 over 12 weeks duration is shown in Fig. 3. This complex plot shows different activities in different grey shaded colour.

In Fig. 4, the Boxplots of worker \#1, \#2 and \#4 representing their office occupancy and computer usage are illustrated. These clearly show the problems associated with categorising and profiling different aspects of a worker's ADW. Although worker \#1 and worker \#2 have similar relationships between their office occupancy and computer usage, worker \#4 exhibits a very different relationship between these aspects of their ADW. Thus a simplistic statistical model would not be sufficient to capture the richness of individual behaviours.

Although Fig. 4 shows that individual users can show markedly different start times and computer usage, the data can be explored further in order to gain a more complete profile of the individuals. Fig. 5 shows three different aspects of a single worker represented by spider (radar) plots. In Fig. 5-a), the data is shown for a given week, where the axes represent the days of the working week, and in Fig 5-b) the axes represent the data averaged over 5 consecutive weeks. The use of 5 weeks of data is simply to allow a comparison with the

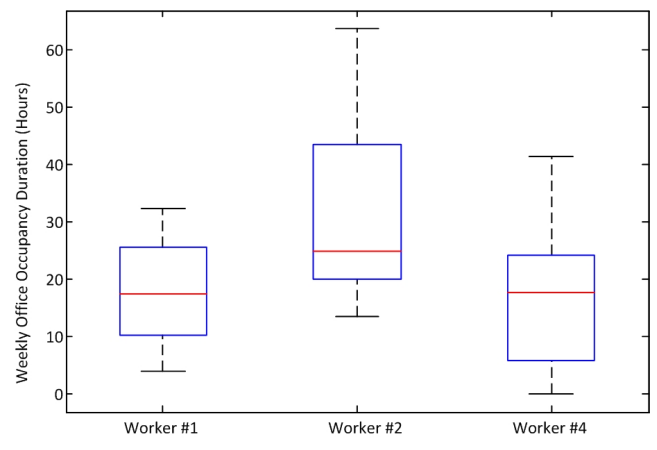

(a) Office occupancy

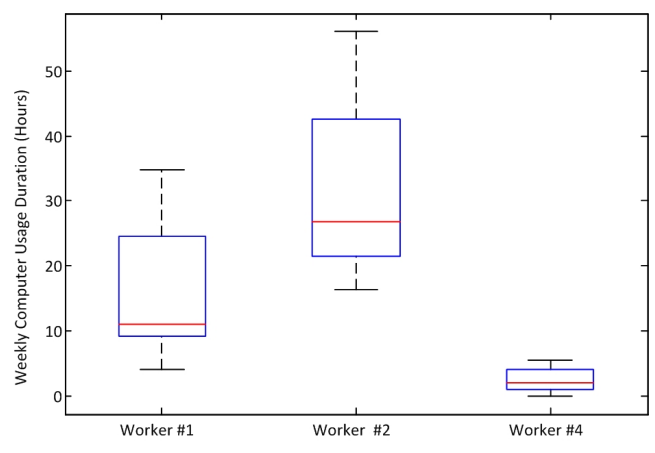

(b) Computer usage

Fig. 4: Box plot representing worker behaviours based on weekly duration.

same angular distribution of axes. It can be seen that the overall office occupancy for the worker varies dramatically from day to day, though it is much more consistent from week to week. This occupancy naturally affects the light usage, which also shows considerable variation from day to day. However, interestingly, despite the variation in occupancy from day to day, the computer usage does not vary as much. This may indicate that the user has a specific amount of computer work to do each day, which takes precedence even when their overall office time is low for that day.

Because these experiments were conducted on academic staff during term time, one should not be surprised that their office occupancy varies dramatically within a working week. Similarly the week to week consistency would also be expected, since teaching duties would be similar each week. However it does indicate that measures used to generate individual worker profiles have to be considered within a multi-scale approach - where the scales relate to time of day, day of week, week of year etc. In that way we can ensure that the model reflects the worker's behaviour more closely and thus use it to try to optimise their office environment more individually. 


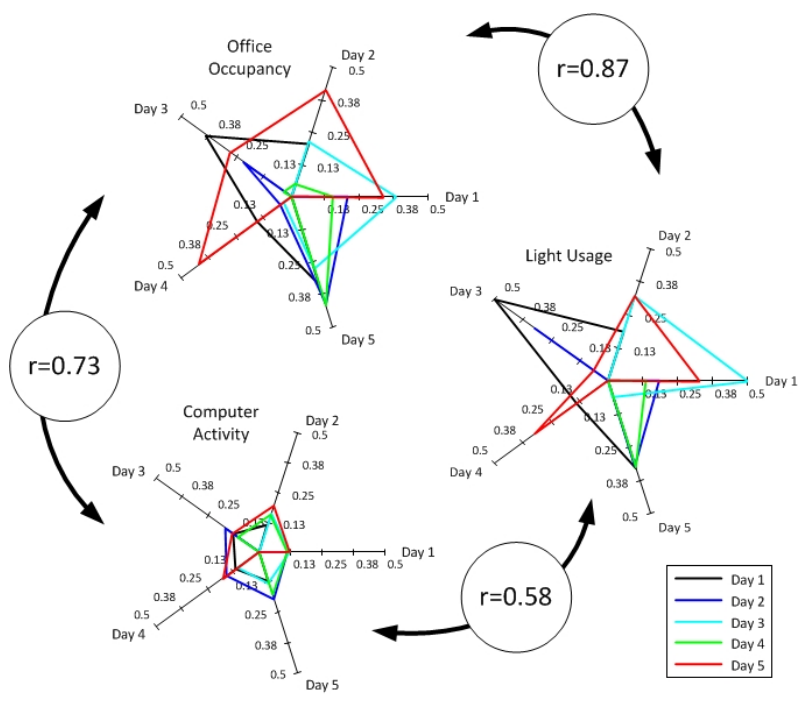

(a) 5 Days

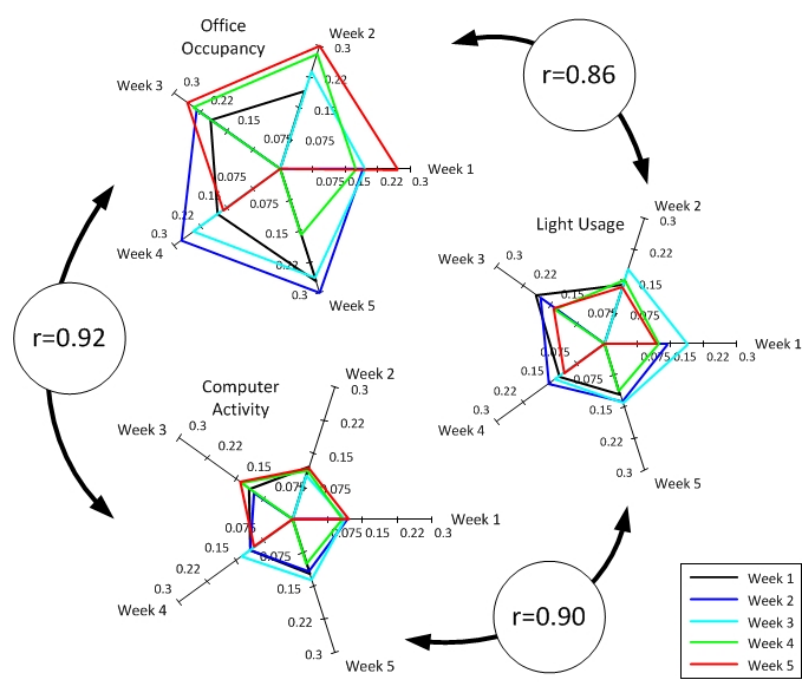

(b) 5 Weeks

Fig. 5: Comparison of variance in activities for worker \#2.

To justify and validate our state model of the office worker activities, the workers we monitored were asked to complete a diary where they recorded their daily actions. These notes were used to generate activity patterns as shown in Fig. 6. For comparison, Fig. 7 shows the same workers' activities as inferred from the raw sensor information. The subjective evidence from the workers clearly matches very well with the states derived from the sensor data, showing that the modelling reflects the worker perceptions and granularity of their activities. If, for example, the workers

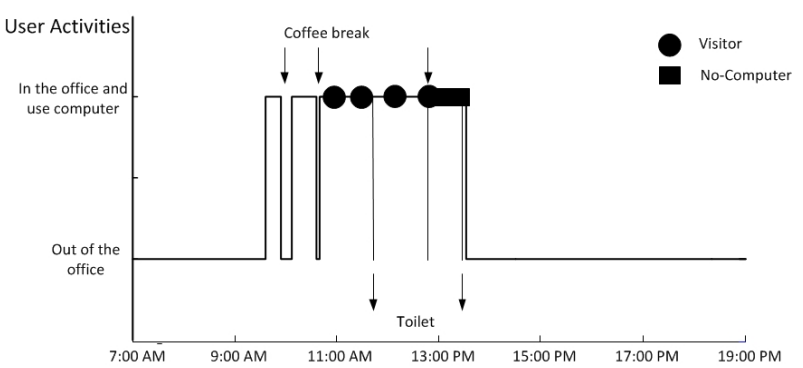

(a) Tuesday, Worker \#2

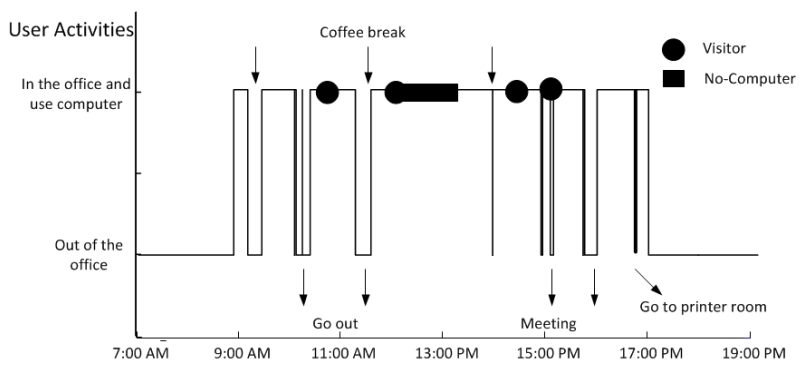

(b) Wednesday, Worker \#2

Fig. 6: Samples of activities patterns based on worker's annotation.

had recorded many fewer out-of-office occasions than inferred from the data, it would show that the workers did not consider these short excursions to be worth noting. Thus a system that (for example) automatically shut down their computer and switched off lights would irritate them if they did not feel they were out of office for very long. However, both the workers and the analysed data matched on these short interludes, indicating that an automatic system would be accepted if it shut down energy consumption for some of these absences.

\section{CONCLUSIONS}

In this paper, we have presented a practical situation where we described the application of fuzzy finite state machines to model a worker's activities in an intelligent office environment. Fuzzy finite state machines are defined for the states of an office worker and way in which they are applied to the modelling of office activities is described in detail. We have shown that although worker behaviour can be represented by fuzzy measures of activities, there are still unsolved problems regarding the mapping of sensor data to the higher levels of activities.

We appreciate that the research presented in this paper is not complete and there are many aspects of the work that could be enhanced. The fuzzy transition function needs to be improved by adding some capabil- 


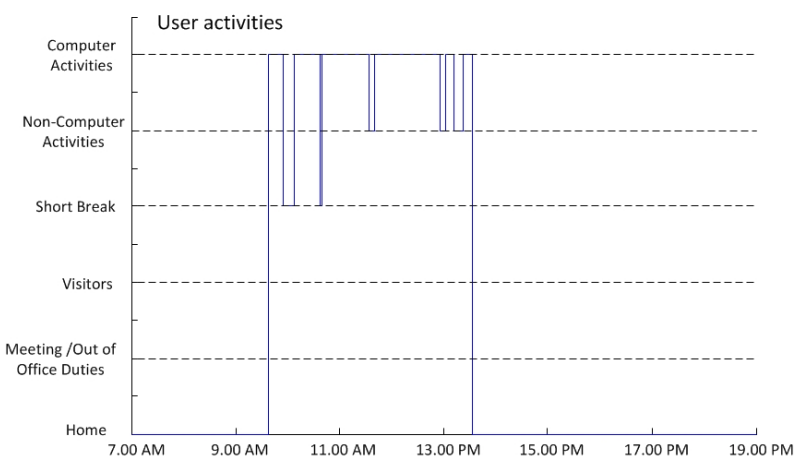

(a) Tuesday, Worker \#2

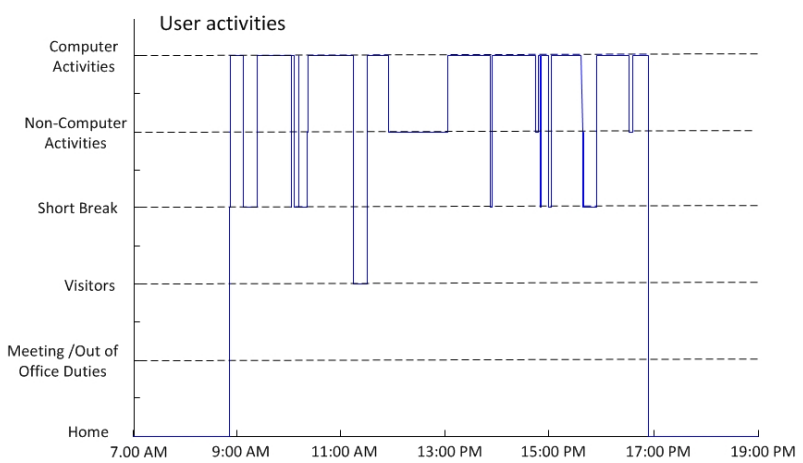

(b) Wednesday, Worker \#2

Fig. 7: Worker's activity model gathered from sensory devices.

ity of learning from data. This could be achieved by incorporating a genetic algorithm or other computational intelligence technique. The second improvement of the work presented here would be to quantify the office worker's comfort and preferences (in terms of office temperature, ventilation etc.) and incorporate these as inputs to the transition function.

\section{REFERENCES}

[1] N. K. Suryadevara, A. Gaddam, R. K. Rayudu, and S. C. Mukhopadhyay, "Wireless sensors network based safe home to care elderly people: Behaviour detection", Sensors and Actuators, vol. 186, pp. 277-283, 102012.

[2] Saifullizam Puteh, Caroline Langensiepen, and Ahmad Lotfi, "Fuzzy ambient intelligence for intelligent office environments", in Proc. of IEEE International Conference on Fuzzy Systems, 2012, pp. 1-6.

[3] Liming Chen, J. Hoey, C. D. Nugent, D. J. Cook, and Zhiwen Yu, "Sensor-based activity recognition", IEEE Trans. on Systems, Man, and Cybernetics, Part C: Applications and Reviews, vol. 42, no. 6, pp. 790-808, 2012.

[4] Vincent Tabak and Bauke de Vries, "Methods for the prediction of intermediate activities by office occupants", Building and Environment, vol. 45, no. 6, pp. 1366-1372, 2010.

[5] Xin Hong and C. D. Nugent, "Partitioning time series sensor data for activity recognition", in Proc. of 9th International Conference on Information Technology and Applications in Biomedicine, 2009, pp. 1-4.
[6] Saisakul Chernbumroong, Shuang Cang, Anthony Atkins, and Hongnian Yu, "Elderly activities recognition and classification for applications in assisted living", Expert Systems with Applications, vol. 40, no. 5, pp. 1662-1674, 42013.

[7] Hamid Medjahed, Dan Istrate, Jerome Boudy, and Bernadette Dorizzi, "Human activities of daily living recognition using fuzzy logic for elderly home monitoring", in Proc. of the International Conference on Fuzzy Systems, Republic of Korea, August 2009, pp. 2001-2006.

[8] D. Naranjo-Hernandez, L. M. Roa, J. Reina-Tosina, and M. A. Estudillo-Valderrama, "Som: A smart sensor for human activity monitoring and assisted healthy ageing", IEEE Transactions on Biomedical Engineering, vol. 59, no. 11, pp. 3177-3184, 2012.

[9] R. R. Fletcher et al, "icalm: Wearable sensor and network architecture for wirelessly communicating and logging autonomic activity", Information Technology in Biomedicine, IEEE Transactions on, vol. 14, no. 2, pp. 215-223, 2010.

[10] Venet Osmani, Sasitharan Balasubramaniam, and Dmitri Botvich, "Human activity recognition in pervasive health-care: Supporting efficient remote collaboration", Journal of Network and Computer Applications, vol. 31, no. 4, pp. 628-655, 11 2008.

[11] P. Rashidi, D.J. Cook, L.B. Holder, and M. SchmitterEdgecombe, "Discovering activities to recognize and track in a smart environment", IEEE Transactions on Knowledge and Data Engineering, vol. 23, no. 4, pp. 527 -539, april 2011.

[12] M. Ros, M. Delgado, and A. Vila, "A system to supervise behaviours using temporal and sensor information", in Proc. of IEEE Int. Conf. on Fuzzy Systems, 2010, pp. 1-8.

[13] H. Madokoro, K. Honma, and K. Sato, "Classification of behavior patterns with trajectory analysis used for event site", in Proc. of International Joint Conference on Neural Networks (IJCNN), 2012, pp. 1-8.

[14] T. Martin, B. Majeed, Beum-Seuk Lee, and N. Clarke, "Fuzzy ambient intelligence for next generation telecare", in Proc. of IEEE International Conference on Fuzzy Systems, 2006, pp. 894-901.

[15] B. S. Lee, T. P. Martin, N. P. Clarke, B. Majeed, and D. Nauck, "Dynamic daily-living patterns and association analyses in telecare systems", in Proc. of the 4th IEEE Int. Conf. on Data Mining (ICDM), 2004, pp. 447-450.

[16] M. Ahad, J. K. Tan, H. S. Kim, and S. Ishikawa, "Human activity recognition: Various paradigms", in Proc. of Int. Conf. on Control, Automation and Systems (ICCAS), 2008, pp. 18961901.

[17] Sung-Ihk Yang and Sung-Bae Cho, "Recognizing human activities from accelerometer and physiological sensors", in Proc. of IEEE Int. Conf. on Multisensor Fusion and Integration for Intelligent Systems (MFI), 2008, pp. 100-105.

[18] Zhelong Wang, Ming Jiang, Yaohua Hu, and Hongyi Li, "An incremental learning method based on probabilistic neural networks and adjustable fuzzy clustering for human activity recognition by using wearable sensors", IEEE Trans. on Information Tech. in Biomedicine, vol. 16, no. 4, pp. 691-699, 2012.

[19] L.M. Reyneri, "An introduction to fuzzy state automata", in Biological and Artificial Computation: From Neuroscience to Technology, Jos Mira, Roberto Moreno-Daz, and Joan Cabestany, Eds., vol. 1240 of Lecture Notes in Computer Science, pp. 273283. Springer Berlin Heidelberg, 1997.

[20] A. Alvarez-Alvarez, G. Trivino, and O. Cordon, "Human gait modeling using a genetic fuzzy finite state machine", IEEE Transactions on Fuzzy Systems, vol. 20, no. 2, pp. 205-223, 2012.

[21] S. Puteh, C. Langensiepen, and A. Lotfi, "Fuzzy ambient intelligence for intelligent office environments", in Proc. of IEEE Int. Conf. on Fuzzy Systems, 2012, pp. 1-6.

[22] Kim Eunju, S. Helal, and D. Cook, "Human activity recognition and pattern discovery", IEEE Pervasive Computing, vol. 9, no. 1 , pp. 48-53, 2010. 